\title{
Scalar fields in nuclear matter: The roles of spontaneous chiral symmetry breaking and nucleon structure
}

\author{
G. Chanfray \\ IPN Lyon, Université de Lyon, Univ. Lyon 1, CNRS/IN2P3, UMR5822, F-69622 Villeurbanne Cedex, France \\ M. Ericson \\ IPN Lyon, Université de Lyon, Univ. Lyon 1, CNRS/IN2P3, UMR5822, F-69622 Villeurbanne Cedex, France and Theory Division, \\ CERN, CH-12111 Geneva, Switzerland
}

(Received 29 October 2010; published 31 January 2011)

\begin{abstract}
Chiral theories with spontaneous symmetry breaking such as the Nambu-Jona-Lasinio (NJL) model lead to the existence of a scalar mode. We present in a detailed manner how the corresponding low-momentum effective Lagrangian involving the scalar field can be constructed starting from the NJL model. We discuss the relevance of the scalar mode for the problem of nuclear binding and saturation. We show that it depends on the nucleon mass origin with two extreme cases. If this origin is entirely due to confinement, the coupling of this mode to the nucleons vanishes, making it irrelevant for the nuclear binding problem. If instead it is entirely due to spontaneous symmetry breaking, it couples to the nucleons but nuclear matter collapses. It is only in the case of a mixed origin with spontaneous breaking that nuclear matter can be stable and reach saturation. We describe models of nucleon structure where this balance is achieved. We also show how chiral constraints and confinement modify the QCD sum rules for mass evolution in nuclear matter.
\end{abstract}

DOI: 10.1103/PhysRevC.83.015204

PACS number(s): 24.85.+p, 11.30.Rd, 12.40.Yx, 13.75.Cs

\section{INTRODUCTION}

The relation between the fundamental properties of lowenergy QCD, namely, chiral symmetry and confinement, and the nuclear many-body problem is one of the most challenging aspects of present-day nuclear physics. One question is how the interplay between chiral symmetry and confinement in the nucleon structure manifests itself in the nuclear many-body problem. In a set of recent papers [1-3], we have associated the mean-field nuclear attraction with the in-medium modification of a (chiral invariant) background scalar field which reflects part of the evolution of the chiral quark condensate. In this framework the nuclear medium can be seen as a shifted QCD vacuum. Nuclear stability is ensured with the phenomenological incorporation of the nucleon response to this scalar field. This response depends on the quark confinement mechanism inside the nucleon. This framework has been implemented in nuclear matter calculation at the Hartree level [1]. In a subsequent work [2], we also incorporated nonrelativistically the pion loop correlation energy. A full relativistic HartreeFock (RHF) calculation was then done in Ref. [3], allowing us to reproduce also the asymmetry properties of nuclear matter.

The aim of this paper is to discuss the foundations of this picture and the nature of this scalar background field. Although the concept of a scalar field has been widely used for nuclear matter studies [4], its precise origin or meaning is still a controversial subject. The problem is that there is no sharp scalar resonance which would lead to a simple scalar particle exchange. In our approach instead we stress the chiral aspect of the problem. As soon as we start from a model which gives a correct description of chiral symmetry breaking in the QCD vacuum such as the Nambu-Jona-Lasinio model (NJL), the emergence of a scalar field linked to the quark condensate cannot be avoided. This is by construction a low-momentum concept which does not imply the existence of a sharp scalar meson if the effect of confinement is taken into account. Indeed it has been demonstrated by Celenza et al. $[5,6]$ that the inclusion of a confining interaction on top of the NJL model pushes the $q \bar{q}$ scalar state, located originally at twice the constituent quark mass, well above $1 \mathrm{GeV}$. As for the $f_{0}(600)$, it appears as a broad $\pi \pi$ resonance which has no direct relation with the scalar field. The explicit construction of the scalar field can be done using a bosonization technique based on a derivative expansion valid at low (spacelike) momenta. The corresponding "scalar mass," which is around twice the constituent quark mass, is in a fact a low-momentum parameter related to the inverse of the vacuum scalar susceptibility. We recall for completeness that, according to Refs. [5,6], the confining interaction has little influence on the low-momentum parameters entering the effective Lagrangian.

A priori, the range of this mass and the magnitude of the scalar coupling to the nucleon make it relevant for nuclear physics. The real question for this relevance is intimately related to the problem of the structure of the nucleon and the origin of its mass. The respective roles of spontaneous symmetry breaking and confinement in the generation of this mass are indeed crucial. Confinement has little effect on the low-momentum parameters, but it leads to the concept of a nucleonic response to the scalar field, as was originally introduced by Guichon [7]. Without it (i.e., in the pure NJL model), nuclear matter would not be stable and would collapse [8] due to attractive three-body forces (tadpole diagram). On the other hand, if the nucleon mass were entirely due to confinement, as in the MIT bag model, the background scalar field of the NJL model would be irrelevant in nuclear physics, since its coupling to the nucleon would vanish. The reason is very simple: the quarks inside the bag, a bubble of perturbative 
vacuum, do not feel the presence of the surrounding scalar field. Said differently, the constituent quarks to which this scalar field couples are in this case absent in the nucleon. It is likely that the nucleon mass has a mixed origin, in part from chiral symmetry breaking and in part from confinement. In this case, the nucleon mass in the nuclear medium can feel the presence of the scalar field of the NJL model. At the same time, it reacts against this field, and it is possible to stabilize nuclear matter. Nuclear saturation may then result from a delicate balance between the influence of chiral symmetry breaking and confinement in nucleon structure. It is clear, however, that the importance of the role played by the background scalar field in nuclear binding and saturation should not be left to prejudice and beliefs but to facts which may help elucidate this role.

This is among the purposes of this article. Some questions to be answered are, If the scalar field is an actor in the nuclear binding and saturation problem, is this role quantitatively compatible with nuclear phenomenology? What information do we have on the role played by confinement? Is it compatible with acceptable models of the nucleon structure? The last question is a motivation for the second part of this work, in which we propose models of the nucleon where confinement and chiral symmetry breaking contribute roughly equally to the mass. The influence of the spontaneous breaking of chiral symmetry is large enough for the background scalar field to act as a source of nuclear attraction. But the confining aspect is sufficient to stabilize nuclear matter.

Our article is organized as follows. Section II is devoted to the Nambu-Jona-Lasinio model. After a brief reminder of the basic properties of the model, we derive from it an effective Lagrangian which is valid for low (spacelike) momenta relevant to nuclear physics studies. We first use a sharp momentum cutoff, and in a final step for practical calculations we use a delocalized version. We conclude that section by some general comments concerning in particular the evolution of the nucleon mass and quark condensate when confinement effects at the level of the nucleon structure are incorporated. In Sec. III, based on a simple quark-diquark string model, we discuss how the interplay between chiral symmetry breaking and confinement in the nucleon structure influences nuclear matter binding properties. We also discuss the influence of the modeling of the confinement mechanism inside the nucleon.

\section{SCALAR BACKGROUND FIELD FROM THE NAMBU-JONA-LASINIO MODEL}

\section{A. Standard NJL model}

We first introduce the NJL model in the light quark sector whose original aim is to describe the low-mass mesons: the $\pi$, $\sigma, \rho, a_{1}$, and $\omega$ mesons. The Lagrangian is

$$
\begin{aligned}
\mathcal{L}= & \bar{\psi}\left(i \gamma^{\mu} \partial_{\mu}-m\right) \psi+\frac{G_{1}}{2}\left[(\bar{\psi} \psi)^{2}+\left(\bar{\psi} i \gamma_{5} \vec{\tau} \psi\right)^{2}\right] \\
& -\frac{G_{2}}{2}\left[\left(\bar{\psi} \gamma^{\mu} \vec{\tau} \psi\right)^{2}+\left(\bar{\psi} \gamma^{\mu} \gamma_{5} \vec{\tau} \psi\right)^{2}+\left(\bar{\psi} \gamma^{\mu} \psi\right)^{2}\right] .
\end{aligned}
$$

Using path integral techniques it can be equivalently written in a semibosonized form

$$
\begin{aligned}
\mathcal{L}= & \bar{\psi}\left[i \gamma^{\mu} \partial_{\mu}-m-\Sigma-i P \gamma^{5}-\gamma^{\mu}\left(\tilde{V}_{\mu}+\gamma_{5} \tilde{A}_{\mu}\right)\right] \psi \\
& -\frac{1}{4 G_{1}} \operatorname{tr}_{f}\left(\Sigma^{2}+P^{2}\right)+\frac{1}{4 G_{2}} \operatorname{tr}_{f}\left(\tilde{V}^{\mu} \tilde{V}_{\mu}+\tilde{A}^{\mu} \tilde{A}_{\mu}\right) .
\end{aligned}
$$

Here $\psi$ represents an isodoublet of quark fields, $\Sigma$ is a scalarisoscalar field, and the matrix $P=\vec{\tau} \cdot \vec{P} \equiv \tau_{j} P_{j}$ describes a pseudoscalar isovector field. The matrix $\tilde{V}^{\mu}=\tilde{\Omega}^{\mu}+\tau_{j} \tilde{V}_{j}^{\mu}$ contains an isoscalar $(\tilde{\Omega})$ and an isovector $\left(\tilde{V}_{j}\right)$ vector field, and $\tilde{A}^{\mu}=\tau_{j} \cdot \tilde{A}_{j}^{\mu}$ is an isovector axial-vector field. The current quark mass is $m$, and $G_{1}$ and $G_{2}$ are two (positive) coupling constants. $\vec{P}$ is the chiral partner of the $\Sigma, \tilde{A}_{j}$ is the chiral partner of $\tilde{V}_{j}$, and in the limit of vanishing $m$ (chiral limit) this Lagrangian is chiral invariant. Coupling to left $\left(\mathcal{L}^{\mu}\right)$ and right $\left(\mathcal{R}^{\mu}\right)$ electroweak currents is included through the replacement

$$
\begin{gathered}
\tilde{V}_{\mu}+\gamma_{5} \tilde{A}_{\mu} \rightarrow \tilde{V}_{\mu}+\gamma_{5} \tilde{A}_{\mu}+\mathcal{V}_{\mu}+\gamma_{5} \mathcal{A}_{\mu} \\
\text { with } \quad \mathcal{V}^{\mu}=\frac{\mathcal{R}^{\mu}+\mathcal{L}^{\mu}}{2}, \quad \mathcal{A}^{\mu}=\frac{\mathcal{R}^{\mu}-\mathcal{L}^{\mu}}{2} .
\end{gathered}
$$

In the mean field approximation, the constituent quark mass $M_{0}$ is the solution of the gap equation

$$
\begin{aligned}
M_{0} & =m+4 N_{c} N_{f} G_{1} M_{0} I_{1}\left(M_{0}\right) \\
\text { with } \quad I_{1} & =\int \frac{i d^{4} k}{(2 \pi)^{4}} \frac{1}{k^{2}-M_{0}^{2}}=\int_{0}^{\Lambda} \frac{d^{3} k}{(2 \pi)^{3}} \frac{1}{2 E_{k}},
\end{aligned}
$$

where $N_{c}=3$ and $N_{f}=2$ are the number of colors and flavors, and $E_{k}=\sqrt{k^{2}+M_{0}^{2}}$. The quark condensate (per flavor) is $\langle\bar{q} q\rangle=-4 N_{c} M_{0} I_{1}$. The second form of the $I_{1}$ integral has been obtained through the introduction of a sharp three-momentum cutoff $\Lambda$. This sharp noncovariant cutoff is first taken for simplicity. We will use later a delocalized version of the NJL which corresponds to a softer cutoff procedure. The mesons can be generated as collective $q \bar{q}$ modes either by applying a standard random-phase approximation to the original Lagrangian or by performing a second-order expansion in the fluctuating fields of the bosonized effective action. We list here some results, and for that purpose we introduce the integral

$$
\begin{aligned}
I(\omega) & \equiv 2 N_{c} N_{f} I_{2}(\omega) \\
& =2 N_{c} N_{f} \int_{0}^{\Lambda} \frac{d^{3} k}{(2 \pi)^{3}} \frac{1}{E_{k}\left(4 E_{k}^{2}-\omega^{2}\right)} .
\end{aligned}
$$

The $q \bar{q}$ scattering amplitude at zero c.m. momentum, $\mathcal{M}^{(\pi)}(\omega)$, in the pion channel is obtained from the polarization bubble $\tilde{\Pi}_{\mathrm{PS}}^{0}(\omega)$ in the pseudoscalar channel incorporating the $\pi-a_{1}$ mixing:

$$
\begin{aligned}
\mathcal{M}^{(\pi)}(\omega) & =\frac{G_{1}}{1-G_{1} \tilde{\Pi}_{\mathrm{PS}}^{0}(\omega)} \equiv \frac{1 / \tilde{I}(\omega)}{\omega^{2}-M_{\pi}^{2}(\omega)} \\
\text { with } \quad M_{\pi}^{2}(\omega) & =\frac{m}{G_{1} M_{0} \tilde{I}(\omega)} .
\end{aligned}
$$

The difference between $\tilde{I}(\omega)=I(\omega) /\left[1+4 M_{0}^{2} G_{2} I(\omega)\right]$ and $I(\omega)$ comes from the mixing effect. From this result, we deduce the physical pion mass, the pion-quark coupling constant, and 
the pion decay constant:

$$
m_{\pi}^{2}=M_{\pi}^{2}\left(m_{\pi}\right), \quad g_{\pi q q}^{2}=\frac{r_{\pi}}{\tilde{I}\left(m_{\pi}\right)}, \quad f_{\pi}^{2}=M_{0}^{2} \tilde{I}\left(m_{\pi}\right) r_{\pi} .
$$

Here the factor $r_{\pi}=\left[1+\frac{m_{\pi}^{2}}{\tilde{I}\left(m_{\pi}\right)}\left(\frac{\partial \tilde{I}}{\partial \omega^{2}}\right)\right]^{-1}$, which in practice is very close to unity, is the residue at the pion pole.

\section{B. Effective theory for low-momentum nuclear physics}

The meson spectrum (scalar and vector mesons) can in principle be obtained in the previous scheme. This approach is notoriously unsatisfactory due to the lack of confinement: in particular, unphysical decay channels of vector mesons in $q \bar{q}$ pairs may appear; but, as discussed in the Introduction, we aim to derive an effective low-momentum theory (i.e., for low spacelike momenta relevant in nuclear physics) and not to discuss the on-shell properties of scalar and vector mesons, in particular their physical masses. Hence our resulting mass parameters for scalar and vector mesons will not be the on-shell masses but simply mass parameters associated with the inverse of the corresponding correlators taken at zero momentum. As emphasized by Celenza et al. [5,6], confinement, which is needed to prevent unphysical decays of mesons, plays a minor role for the low-momentum fields (in particular the scalar one) relevant in nuclear physics. We now describe the technical steps needed to establish the form of the effective low-momentum Lagrangian.

\section{Effective Lagrangian from NJL model}

The aim is to establish a low-momentum Lagrangian in the meson sector so as to generate the dynamics of the scalar field. Technically this can be done by integrating out quarks in the Dirac sea using a path integral formalism. The physical meaning is simply a projection of $q \bar{q}$ vacuum fluctuations onto mesonic degrees of freedom.

In the spirit of our previous works $[1-3,9,10]$, we first go from the Cartesian representation $(\Sigma, P)$ to a polar representation $(\mathcal{S}, \pi)$ by making the change of variables:

$$
m+\Sigma+i P=\mathcal{S} U \text { with } U \equiv \xi^{2}=e^{i \vec{\tau} \cdot \vec{\pi}} .
$$

The motivation is that the description of nuclear matter attraction is more suited as explained in particular in Ref. [10]. It is convenient to introduce a new quark field $q$ defined by

$$
q=\xi_{5} \psi, \quad \bar{q}=\bar{\psi} \xi_{5}, \quad \text { with } \quad \xi_{5}=e^{i \vec{\tau} \cdot \vec{\pi} \gamma_{5} / 2},
$$

and new vector and axial-vector fields according to

$$
\begin{aligned}
& V^{\mu}=\xi \frac{\tilde{V^{\mu}}+\tilde{A^{\mu}}}{2} \xi^{\dagger}+\xi^{\dagger} \frac{\tilde{V^{\mu}}-\tilde{A^{\mu}}}{2} \xi-\mathcal{V}_{c}^{\mu}+\mathcal{V}_{\xi}^{\mu}, \\
& A^{\mu}=\xi \frac{\tilde{V^{\mu}}+\tilde{A^{\mu}}}{2} \xi^{\dagger}-\xi^{\dagger} \frac{\tilde{V^{\mu}}-\tilde{A^{\mu}}}{2} \xi-\mathcal{A}_{c}^{\mu}+\mathcal{A}_{\xi}^{\mu},
\end{aligned}
$$

with

$$
\begin{array}{ll}
\mathcal{V}_{c}^{\mu}=\frac{i}{2}\left(\xi \partial^{\mu} \xi^{\dagger}+\xi^{\dagger} \partial^{\mu} \xi\right), & \mathcal{A}_{c}^{\mu}=\frac{i}{2}\left(\xi \partial^{\mu} \xi^{\dagger}-\xi^{\dagger} \partial^{\mu} \xi\right) \\
\mathcal{V}_{\xi}^{\mu}=\frac{1}{2}\left(\xi \mathcal{R}^{\mu} \xi^{\dagger}+\xi^{\dagger} \mathcal{L}^{\mu} \xi\right), & \mathcal{A}_{\xi}^{\mu}=\frac{1}{2}\left(\xi \mathcal{R}^{\mu} \xi^{\dagger}-\xi^{\dagger} \mathcal{L}^{\mu} \xi\right)
\end{array}
$$

There is in principle a Jacobian associated with the above Weinberg transformation which is usually taken into account in the functional integral by introducing ghost fields. This point is discussed in Ref. [11], but we do not consider it in this paper. With the new fields defined in Eqs. (7)-(9), the semibosonized Lagrangian takes the form

$$
\begin{aligned}
\mathcal{L}= & \bar{q}\left[i \gamma^{\mu} \partial_{\mu}-\mathcal{S}-\gamma^{\mu}\left(V_{\mu}+\gamma_{5} A_{\mu}\right)\right] q \\
& -\frac{1}{4 G_{1}} \operatorname{tr}_{f}\left[\mathcal{S}^{2}-m \mathcal{S}\left(U+U^{\dagger}\right)\right] \\
& +\frac{1}{4 G_{2}} \operatorname{tr}_{f}\left[\left(V^{\mu}+\mathcal{V}_{c}^{\mu}-\mathcal{V}_{\xi}^{\mu}\right)^{2}+\left(A^{\mu}+\mathcal{A}_{c}^{\mu}-\mathcal{A}_{\xi}^{\mu}\right)^{2}\right]
\end{aligned}
$$

The next step is to integrate out the quarks in the Dirac sea. In that way the kinetic energy term of the mesons fields will be dynamically generated from the quarks loops, i.e, from quantum fluctuations. For convenience, we go from Minkowski space to Euclidean space. Using standard transformation rules, the corresponding Euclidean Lagrangian is

$$
\begin{aligned}
& L_{E}=\bar{q} D q \quad \text { with } \quad D=i \gamma_{\mu}^{E} \cdot \Pi_{\mu}+\mathcal{S}, \\
& \Pi^{\mu}=P_{\mu}-\Gamma_{\mu} \equiv-i \partial_{\mu}-\left(V_{\mu}+\gamma_{5} A_{\mu}\right) .
\end{aligned}
$$

The Euclidean partition function is expressed in terms of the fermion determinant according to

$$
Z=e^{-S_{F}}=\int d q d \bar{q} e^{-d^{4} x \bar{q} D q} .
$$

Ignoring its imaginary part, the effective action can be written as

$$
\begin{aligned}
S_{F} & =-\operatorname{Tr} \ln D=-\frac{1}{2} \operatorname{Tr} \ln \left(D D^{\dagger}\right) \\
& =-\frac{1}{2} \operatorname{Tr} \ln \left(\Pi_{+}^{2} i \alpha_{\mu \nu} W_{\mu \nu}+\mathcal{S}^{2}-\gamma_{\mu} \mathcal{D}_{\mu} \mathcal{S}\right)
\end{aligned}
$$

with

$$
\begin{aligned}
\Pi_{\mu} & =P_{\mu}-\Gamma_{\mu} \equiv-i \partial_{\mu}-\left(V_{\mu}+\gamma_{5} A_{\mu}\right), \\
\mathcal{D}_{\mu} \mathcal{S} & =\partial_{\mu} \mathcal{S}+2 i \gamma_{5} A_{\mu}, \\
W_{\mu \nu} & =\partial_{\mu} \Gamma_{\nu}-\partial_{\nu} \Gamma_{\mu}-i\left[\Gamma_{\mu}, \Gamma_{\nu}\right] .
\end{aligned}
$$

We perform a derivative expansion valid at low momentum of the fermion determinant to second order in the derivatives. The difficulty lies in the fact that we do not make an expansion around a constant (vacuum expectation value of the scalar field), but we want to have a formal expansion with the scalar objet $\mathcal{S}$ keeping its field status, so as to include its possible modification in the nuclear environment. For that purpose, we use the elegant method proposed by Chan [12]. The starting point is the following trick which uses translational invariance in momentum space:

$$
\begin{aligned}
\operatorname{Tr}\left[A\left(\Pi_{\mu}, G(X)\right)\right] & =\operatorname{Tr}\left[e^{i k \cdot X} A\left(\Pi_{\mu}, G(X)\right) e^{-i k \cdot X}\right] \\
& =\operatorname{Tr} A\left(\Pi_{\mu}+k_{\mu}, G(X)\right),
\end{aligned}
$$

where $A$ represents any operator depending of the position operator $X$ and of the generalized momentum $\Pi$. Hence the 
arbitrary four-momentum $k$ can be averaged:

$$
\begin{aligned}
\operatorname{Tr} & {\left[A\left(\Pi_{\mu}, G(X)\right)\right] } \\
& =\frac{1}{\delta^{(4)}(0)} \int \frac{d^{4} k}{(2 \pi)^{4}} \operatorname{Tr} A\left(\Pi_{\mu}+k_{\mu}, G(X)\right) .
\end{aligned}
$$

It follows that the quark determinant can be calculated as

$$
\begin{aligned}
S_{F}= & -\frac{1}{2} \frac{1}{\delta^{(4)}(0)} \int \frac{d^{4} k}{(2 \pi)^{4}} \operatorname{Tr}\left[\ln \left(G^{-1}\right)\right. \\
& \left.+\ln \left(1+G \Pi^{2}+G(2 k \cdot \Pi+a)\right)\right], \\
& \text { with } \quad G=\left(k^{2}+\mathcal{S}^{2}\right)^{-1}, \quad a=i \alpha_{\mu \nu} W_{\mu \nu}-\gamma_{\mu} \mathcal{D}_{\mu} \mathcal{S} .
\end{aligned}
$$

As pointed out by Chan, the introduction of the momentum integration does not disturb the full trace operation and offers the freedom needed for manipulations, such as cyclic permutations of the operators or integrations by part under the condition that there is an implicit regularization scheme. The essential point is that the final form for the action is entirely expressible in terms of covariant derivatives $\left[\Pi_{\mu}, \mathcal{S}\right]$ and $\left[\Pi_{\mu}, \Pi_{\nu}\right]=W_{\mu \nu}$, as it should. Once this is done, the explicit trace over $|x\rangle$ states can be performed, producing the $\delta^{(4)}(0)$ compensating the one coming from the average procedure. To perform a second-order derivative expansion corresponds in practice to make a fourth-order expansion in $\Pi_{\mu}$ and second order in $a$. The result is

$$
\begin{aligned}
S_{F}= & -\frac{1}{2} \int \frac{d^{4} k d^{4} x}{(2 \pi)^{4}} \operatorname{tr}_{\mathrm{DFC}}\left(\ln \left(G^{-1}\right)-\frac{1}{2} G^{2} a^{2}\right. \\
& \left.+k^{2} G^{2} \mathcal{S}^{2} \partial_{\mu} \mathcal{S} \partial_{\mu} \mathcal{S}+\frac{k^{2} k^{2}}{12} W_{\mu \nu} W_{\mu \nu}\right),
\end{aligned}
$$

where the trace operation acts in Dirac, flavor, and color spaces. The resulting effective action is reducible to a local Lagrangian. Coming back to Minkowski space but keeping the momentum $k$ explicitly in Euclidean space, this local Lagrangian $\mathcal{L}_{\text {mes }}$ has the form

$$
\begin{aligned}
\mathcal{L}_{\text {mes }}= & \frac{1}{2} 2 N_{c} N_{f} I_{2 S}(\mathcal{S}) \partial^{\mu} \mathcal{S} \partial_{\mu} \mathcal{S}-W(\mathcal{S}) \\
& +\frac{m \mathcal{S}}{4 G_{1}} \operatorname{tr}_{f}\left(U+U^{\dagger}-2\right)+\frac{1}{2} 2 N_{c} N_{f} I_{2}(\mathcal{S}) 4 \vec{A}^{\mu} \cdot \vec{A}_{\mu} \mathcal{S}^{2} \\
& +\frac{1}{4 G_{2}} \operatorname{tr}_{f}\left[\left(V^{\mu}+\mathcal{V}_{c}^{\mu}-\mathcal{V}_{\xi}^{\mu}\right)^{2}+\left(A^{\mu}+\mathcal{A}_{c}^{\mu}-\mathcal{A}_{\xi}^{\mu}\right)^{2}\right] \\
& -\frac{1}{6} 2 N_{c} N_{f} I_{2 V}(\mathcal{S})\left(\Omega^{\mu \nu} \Omega_{\mu \nu}+\vec{V}^{\mu \nu} \cdot \vec{V}_{\mu \nu}+\vec{A}^{\mu \nu} \cdot \vec{A}_{\mu \nu}\right) .
\end{aligned}
$$

The chiral effective potential $W(\mathcal{S})$ is

$$
W(\mathcal{S})=-2 N_{c} N_{f} I_{0}(\mathcal{S})+\frac{(\mathcal{S}-m)^{2}}{2 G_{1}} .
$$

The quantity $-I_{0}(\mathcal{S})$ represents the vacuum energy density per degrees of freedom associated with the Dirac sea

$$
\begin{aligned}
I_{0}(\mathcal{S}) & =\int \frac{i d^{4} k_{E}}{(2 \pi)^{4}} \ln \left(k_{E}^{2}+\mathcal{S}^{2}\right)=\int_{0}^{\Lambda} \frac{d^{3} k}{(2 \pi)^{3}} E_{k}(\mathcal{S}), \\
E_{k} & \equiv E_{k}(\mathcal{S})=\sqrt{k^{2}+\mathcal{S}^{2}}
\end{aligned}
$$

where the second form corresponds to the sharp noncovariant cutoff. The integral $I_{2}(\mathcal{S})$ is the usual NJL loop integral

$$
I_{2}(\mathcal{S})=\int \frac{i d^{4} k_{E}}{(2 \pi)^{4}} \frac{1}{\left(k_{E}^{2}+\mathcal{S}^{2}\right)^{2}}=\int_{0}^{\Lambda} \frac{d^{3} k}{(2 \pi)^{3}} \frac{1}{4 E_{k}^{3}(\mathcal{S})} .
$$

The integrals $I_{2 S, V}(\mathcal{S})$ entering the scalar and vector kinetic energy terms are

$$
\begin{aligned}
I_{2 S} & =I_{2}-2 \mathcal{S}^{2} I_{3}+2 \mathcal{S}^{4} I_{4}, \quad I_{2 V}=I_{2}+\mathcal{S}^{2} I_{3}-\frac{1}{2} \mathcal{S}^{4} I_{4}, \\
I_{3}(\mathcal{S}) & =\int \frac{i d^{4} k_{E}}{(2 \pi)^{4}} \frac{1}{\left(k_{E}^{2}+\mathcal{S}^{2}\right)^{3}}=\int_{0}^{\Lambda} \frac{d^{3} k}{(2 \pi)^{3}} \frac{3}{16 E_{k}^{5}(\mathcal{S})}, \\
I_{4}(\mathcal{S}) & =\int \frac{i d^{4} k_{E}}{(2 \pi)^{4}} \frac{1}{\left(k_{E}^{2}+\mathcal{S}^{2}\right)^{4}}=\int_{0}^{\Lambda} \frac{d^{3} k}{(2 \pi)^{3}} \frac{5}{96 E_{k}^{7}(\mathcal{S})} .
\end{aligned}
$$

This expression of the Lagrangian shows that our objective of eliminating the vacuum $q \bar{q}$ fluctuations in terms of "observable" background fields is realized. Contrary to usual methods, the integrals $I_{2 S, V}$ appearing in the scalar and vector kinetic energy terms differ from the usual $I_{2}$ integral. However, the difference between $I_{2 S, V}$ and $I_{2}$ has no influence on the nuclear matter calculation, at least in the Hartree approximation, since the derivative terms play no role. It is also important to notice that the derivation has been done assuming implicitly a covariant regularization procedure and may not be strictly valid for the noncovariant cutoff which will be used in practice.

The mesonlike Lagrangian written above in Eq. (20) has been obtained by integrating out the fluctuating quark fields (quarks in the Dirac sea). It remains to enlarge this Lagrangian to the "classical" quark fields corresponding to the valence quark sector. Formally this can be done by introducing quark source terms $(\bar{\omega}, \omega)$ and splitting the quark field $q$ into a classical part $Q$ and a fluctuating part $\eta=q-Q$. For convenience, we use again the Euclidean formulation and introduce the source-dependent partition function:

$$
Z(\bar{\omega}, \omega)=e^{-W(\bar{\omega}, \omega)}=\int d \eta d \bar{\eta} e^{-d^{4} x(\bar{q} D q-\bar{q} \omega-\bar{\omega} q)} .
$$

The exact classical quark field is approximated by the one satisfying the classical equation of motion $D Q=\omega$, and the terms linear in the fluctuation disappear in the exponent. The integration of the fluctuation part produces the above quark determinant, and we obtain for the generating functional

$$
W(\bar{\omega}, \omega)=-\operatorname{Tr} \ln D+\bar{Q} D Q-\bar{Q} \omega-\bar{\omega} Q .
$$

The effective action is obtained by the Legendre transformation:

$$
\Gamma(\bar{Q}, Q)=W(\bar{\omega}, \omega)+\bar{Q} \omega+\bar{\omega} Q=-\operatorname{Tr} \ln D+\bar{Q} D Q .
$$

Hence we recover the fermion determinant containing the meson fields plus a classical piece described by anticommuting fermions fields that we associate with the non-integrated-out valence quarks interacting with the meson fields. Coming back to the Minkowski space, we get in addition to the mesonic Lagrangian, $\mathcal{L}_{\text {mes }}$, the valence quark effective Lagrangian 
which simply reads

$$
\mathcal{L}_{\text {val }}=\bar{Q}\left[i \gamma^{\mu} \partial_{\mu}-\mathcal{S}-\gamma^{\mu}\left(V_{\mu}+\gamma_{5} A_{\mu}\right)\right] Q .
$$

Recall that $\mathcal{S}$ is still a field, and its vacuum expectation value [corresponding to the minimum of $W(\mathcal{S})$ ] is the vacuum constituent quark mass $M_{0}$, solution of the gap equation (3). In nuclear matter, its expectation value $\mathcal{\mathcal { S }}$ is the solution of an in-medium gap equation modified by the presence of the nucleonic scalar density. It coincides with the in-medium modified constituent quark mass $M$ (see the next section). Its fluctuation enters the scalar exchange Fock term according to the treatment given in Ref. [3]. In the following, we will replace in Eq. (20) the scalar field by its expectation value in all derivatives terms and in the extra mass term, $I_{2}(\mathcal{S}) 4 \vec{A}^{\mu} \cdot \vec{A}_{\mu} \mathcal{S}^{2}$, for the axial-vector meson. In order to prepare the identification of canonical modes, we rewrite (omitting electroweak fields) the mesonic Lagrangian as

$$
\begin{aligned}
\mathcal{L}_{\text {mes }}= & \frac{1}{2} 2 N_{c} N_{f} I_{2 S}(\overline{\mathcal{S}}) \partial^{\mu} \mathcal{S} \partial_{\mu} \mathcal{S}-W(\mathcal{S})+\frac{m \mathcal{S}}{4 G_{1}} \operatorname{tr}_{f}\left(U+U^{\dagger}-2\right)+\tilde{I}(\overline{\mathcal{S}}) \overline{\mathcal{S}}^{2} \operatorname{tr}_{f}\left(\mathcal{A}_{c}^{\mu} \cdot \mathcal{A}_{\mu}^{c}\right) \\
& +\frac{1}{4 G_{2}}\left[1+4 G_{2} I(\overline{\mathcal{S}}) \overline{\mathcal{S}}^{2}\right] \operatorname{tr}_{f}\left(A^{\mu}+\frac{\mathcal{A}_{c}^{\mu}}{1+4 G_{2} \overline{\mathcal{S}}^{2} I(\overline{\mathcal{S}})}\right)^{2} \\
& +\frac{1}{4 G_{2}} \operatorname{tr}_{f}\left(V^{\mu}+\mathcal{V}_{c}^{\mu}\right)^{2}-\frac{1}{6} 2 N_{c} N_{f} I_{2 V}(\overline{\mathcal{S}})\left(\Omega^{\mu \nu} \Omega_{\mu \nu}+\vec{V}^{\mu \nu} \cdot \vec{V}_{\mu \nu}+\vec{A}^{\mu \nu} \cdot \vec{A}_{\mu \nu}\right),
\end{aligned}
$$

where we have introduced the quantities

$$
\begin{aligned}
& I(\overline{\mathcal{S}})=2 N_{c} N_{f} I_{2}(\overline{\mathcal{S}}) \\
& \tilde{I}(\overline{\mathcal{S}}) \equiv 2 N_{c} N_{f} \tilde{I}_{2}(\overline{\mathcal{S}})=\frac{I(\overline{\mathcal{S}})}{1+4 G_{2} \overline{\mathcal{S}}^{2} I(\overline{\mathcal{S}})} .
\end{aligned}
$$

We now redefine the axial-vector meson field in order to eliminate the $\pi-a_{1}$ mixing. For this purpose, we introduce the canonical axial-vector field $a_{\mu}$ defined according to

$$
\begin{aligned}
A^{\mu}+\frac{\mathcal{A}_{c}^{\mu}}{1+4 G_{2} \overline{\mathcal{S}}^{2} I(\overline{\mathcal{S}})} & =g_{V} a^{\mu}, \\
\text { with } \quad g_{V}^{2} & =\frac{3 / 2}{2 N_{c} N_{f} I_{2 V}(\overline{\mathcal{S}})},
\end{aligned}
$$

where $g_{V}$ is the quark-vector coupling constant. Similarly the canonical vector $\left(\omega_{\mu}, v_{\mu}\right)$ and scalar $(S)$ fields are defined as

$$
\begin{aligned}
\Omega^{\mu} & =g_{V} \omega^{\mu}, \quad V^{\mu}=g_{V} v^{\mu}, \\
\mathcal{S} & =g_{0 S} S, \quad \text { with } \quad g_{0 S}^{2}=\frac{1}{2 N_{c} N_{f} I_{2 S}\left(M_{0}\right)} .
\end{aligned}
$$

Here the quark-scalar coupling constant $g_{0 S}$ is defined at the vacuum point. Omitting the $\rho \pi \pi$ coupling terms, the lowmomentum effective Lagrangian takes the form

$$
\begin{aligned}
\mathcal{L}_{\text {mes }}= & \frac{1}{2} \frac{I_{2 S}(\overline{\mathcal{S}})}{I_{2 S}\left(M_{0}\right)} \partial^{\mu} S \partial \mu S+W\left(\mathcal{S}=g_{0 S} S\right) \\
& +\frac{m \mathcal{S}}{4 G_{1}} \operatorname{tr}_{f}\left(U+U^{\dagger}-2\right)+\tilde{I}(\overline{\mathcal{S}}) \overline{\mathcal{S}}^{2} \operatorname{tr}_{f}\left(\mathcal{A}_{c}^{\mu} \cdot \mathcal{A}_{\mu}^{c}\right) \\
& +\frac{1}{2} M_{V}^{2}\left(\omega^{\mu} \omega_{\mu}+\vec{v}^{\mu} \cdot \vec{v}_{\mu}\right)+\frac{1}{2} M_{A}^{2}\left(\vec{a}^{\mu} \cdot \vec{a}_{\mu}\right) \\
& -\frac{1}{4}\left(\omega^{\mu \nu} \omega_{\mu \nu}+\vec{v}^{\mu \nu} \cdot \vec{v}_{\mu \nu}+\vec{a}^{\mu \nu} \cdot \vec{a}_{\mu \nu}\right) .
\end{aligned}
$$

The vector and axial-vector low-momentum mass parameters are given by

$$
M_{V}^{2}=\frac{g_{V}^{2}}{G_{2}}, \quad \frac{M_{A}^{2}}{M_{V}^{2}}=1+4 G_{2} I(\overline{\mathcal{S}}) \overline{\mathcal{S}}^{2} .
$$

One defines the canonical pion field, $\Phi \equiv \vec{\tau} \cdot \vec{\Phi}$, through $U=$ $\exp (i \Phi / F)$, where the constant $F$ is given by $F^{2}=\tilde{I}\left(M_{0}\right) M_{0}^{2}$. Coming back to the previous form of the Lagrangian [Eq. (20)], a direct inspection of the coupling of $\partial^{\mu} \Phi$ to the axial weak current allows us to identify the pion decay constant $F_{\pi}$ with the parameter $F$. The vacuum pion mass parameter is finally obtained as

$$
M_{\pi}^{2}=\frac{m M_{0}}{G_{1} F_{\pi}^{2}}
$$

In nuclear matter, the explicit $\mathcal{S}$ factor in front of the pion mass term renormalizes the pion mass. As in Ref. [2], we do not consider this effect. Indeed we know phenomenologically that the pion mass is almost not modified in nuclear matter, since the the pion-nucleon isoscalar scattering length is essentially compatible with zero. Within a chiral approach beyond this present approach, several terms interfere destructively to realize the stability of the pion mass in nuclear matter [13]. As for the (canonical) vacuum scalar mass, it is

$$
\begin{aligned}
M_{\sigma}^{2} & =g_{0 S}^{2}\left(\frac{\partial^{2} W}{\partial \mathcal{S}^{2}}\right)_{\mathcal{S}=M_{0}} \\
& =\frac{I_{2}\left(M_{0}\right)}{I_{2 S}\left(M_{0}\right)}\left[4 M_{0}^{2}+\left(\frac{M_{V}^{2}}{M_{A}^{2}}\right)_{\mathrm{vac}} M_{\pi}^{2}\right] .
\end{aligned}
$$

We stress again that the quantities $M_{V}, M_{A}, M_{\pi}, F_{\pi}$ are not on-shell constants but low-momentum effective Lagrangian parameters, i.e., taken at zero momentum. In practice, however, $M_{\pi}$ and $F_{\pi}$ differ little from the physical pion mass and pion decay constant. On the contrary, the vector and axialvector mass parameters have a priori no reason to coincide with the physical $\omega, \rho$, and $a_{1}$ meson masses. We now develop an alternative approach which relaxes the sharp cutoff procedure. 


\section{Delocalized NJL model}

As seen before, when a vector interaction term is added, the $\pi-a_{1}$ mixing has the effect of decreasing the pion decay constant, and it is not easy with the sharp cutoff to reach a sufficiently large value for $F_{\pi}$. This is one motivation for adopting, for practical phenomenological calculations, another version of the NJL model with a smooth cutoff function. As discussed below, there is also physical motivation for such a smooth regularization associated with nonlocalities.

The nonlocal version of the NJL model is obtained, for any channel, with the replacement

$$
\begin{aligned}
& \left(\bar{\psi} \Gamma_{j} \psi\right)(x) \rightarrow J_{j}(x) \\
& \quad=\int d^{4} x_{1} d^{4} x_{2} F_{c}\left(x_{1}-x\right) F_{c}\left(x-x_{2}\right) \bar{\psi}\left(x_{1}\right) \Gamma_{j} \psi\left(x_{2}\right) .
\end{aligned}
$$

The presence of the form factor automatically provides a regularization procedure. Moreover, such a delocalized Lagrangian possesses a physical basis in terms of a quark-instanton interaction [14]. We define the Fourier transform $f(p)$ of the form factor appearing in the delocalized currents $J_{j}(x)$,

$$
F_{c}(x)=\int \frac{d^{4} p}{(2 \pi)^{4}} e^{-i p \cdot x} f(p),
$$

with $f(0)=1$ in such a way that $F_{c}$ satisfies the normalization condition $\int d^{4} x F_{c}(x)=1$. As can be checked, this procedure maintains the chiral invariance of the interaction. For actual calculations, we will take the noncovariant version of the nonlocal NJL model

$$
F_{c}(x)=\delta(t) F(\vec{r}), \quad f(p) \equiv f(\vec{p})=\int d^{3} r e^{-i \vec{p} \cdot \vec{r}} F(\vec{r}) .
$$

One practical consequence is that the interaction when written in momentum space is modified according to

$$
\begin{aligned}
& G_{j} \delta^{(3)}\left(\vec{p}_{1}+\vec{p}_{2}-\vec{p}_{3}-\vec{p}_{4}\right) \\
& \quad \rightarrow G_{j} \delta^{(3)}\left(\vec{p}_{1}+\vec{p}_{2}-\vec{p}_{3}-\vec{p}_{4}\right) f\left(\vec{p}_{1}\right) f\left(\vec{p}_{2}\right) f\left(\vec{p}_{3}\right) f\left(\vec{p}_{4}\right) .
\end{aligned}
$$

As it is always implicitly done in NJL (with various cutoff prescriptions), we also apply the delocalization procedure to the current quark mass term:

$$
\begin{aligned}
m \bar{\psi}(x) \psi(x) \rightarrow & m \int d^{4} x_{1} d^{4} x_{2} \\
& \times F_{c}\left(x_{1}-x\right) F_{c}\left(x-x_{2}\right) \bar{\psi}\left(x_{1}\right) \psi\left(x_{2}\right) .
\end{aligned}
$$

It can be checked that the QCD realization of explicit chiral symmetry breaking is not affected in the sense that the operator identity $\left[Q_{i}\left[Q_{j}, H\right]\right]=H_{\chi S B}$ is still realized, and consequently the Gell-mann-Oakes-Renner (GOR) relation also holds. In the following, we choose a Gaussian for the form factor

$$
f(\vec{p})=e^{\frac{-p^{2}}{2 \Lambda^{2}}}
$$

where $\Lambda$ (possibly related to the inverse of the instanton size) is the cutoff parameter of the order of $1 \mathrm{GeV}$. One advantage of the nonlocal version is the smooth momentum dependence of the constituent quark mass, in agreement with lattice calculation. Indeed the gap equation in vacuum is written as

$$
\begin{aligned}
M(\vec{p}) & =M_{0} f^{2}(\vec{p}), \quad \text { with } \quad M_{0}=m-2 G_{1}\langle\bar{q} q\rangle, \\
\langle\bar{q} q\rangle & =-N_{c} N_{f} \int \frac{d^{3} k}{(2 \pi)^{3}} f^{2}(\vec{k}) \frac{M(k)}{E_{k}}, \\
E_{k} & =\sqrt{k^{2}+M^{2}(k)} .
\end{aligned}
$$

The delocalized version of the semibosonized form of Eq. (2) is

$$
\begin{aligned}
\mathcal{L}(x)= & \bar{\psi}(x) i \gamma^{\mu} \partial_{\mu} \psi(x)-\int d^{4} x_{1} d^{4} x_{2} \bar{\psi}\left(x_{1}\right) F_{c}\left(x_{1}-x\right)\left[\Sigma+i P \gamma^{5}+\gamma^{\mu}\left(\tilde{V}_{\mu}+\gamma_{5} \tilde{A}_{\mu}\right)\right](x) F_{c}\left(x-x_{2}\right) \psi\left(x_{2}\right) \\
& -\frac{1}{4 G_{1}} \operatorname{tr}_{f}\left((\Sigma-m)^{2}+P^{2}\right)(x)+\frac{1}{4 G_{2}} \operatorname{tr}_{f}\left(\tilde{V}^{\mu} \tilde{V}_{\mu}+\tilde{A}^{\mu} \tilde{A}_{\mu}\right)(x)
\end{aligned}
$$

In the presence of the form factor, we found it more convenient to perform the quark integration with the original quark field. Going again in Euclidean space, the Dirac operator $D^{l}$ is defined according to

$$
\begin{aligned}
D^{l} & =i \gamma_{\mu}^{E} \cdot \Pi_{\mu}^{l}+W^{l}, \quad \Pi_{\mu}^{l}=P_{\mu}-\Gamma_{\mu}^{l}, \\
\Gamma_{\mu}^{l} & =\hat{F}(P)\left(\tilde{V}_{\mu}+\gamma_{5} \tilde{A}_{\mu}\right) \hat{F}(P), \\
W^{l} & =\hat{F}(P) W \hat{F}(P), \quad W=\Sigma+i \gamma_{5} P \equiv \mathcal{S} U_{5} \equiv \mathcal{S} \xi_{5}^{2},
\end{aligned}
$$

where $\hat{F}(P)$ is an operator diagonal in momentum space whose eigenvalues coincide with the form factor $f(p)$. We can see that the effect of nonlocal coupling is to transform all the field operators $O(X)$ into $O^{l}(X)=\hat{F}(P) O(X) \hat{F}(P)$. Inclusion of electroweak coupling is done by making the replacement of the type $\hat{F}(P) \tilde{A}_{\mu} \hat{F}(P) \rightarrow \hat{F}(P) \tilde{A}_{\mu} \hat{F}(P)+\mathcal{A}_{\mu}$.

The Euclidean effective action is

$$
\begin{aligned}
S_{F} & =-\operatorname{Tr} \ln D^{l}=-\frac{1}{2} \operatorname{Tr} \ln \left(D^{l} D^{l \dagger}\right) \\
& =-\frac{1}{2} \operatorname{Tr} \ln \left(\Pi^{l 2}+i \alpha_{\mu \nu} W_{\mu \nu}^{l}+W^{l} W^{l \dagger}-\gamma_{\mu} \mathcal{D}_{\mu}^{l} W^{l}\right),
\end{aligned}
$$

with

$$
\begin{aligned}
\mathcal{D}_{\mu}^{l} W^{l}= & \partial_{\mu} \Sigma^{l}-\left\{\tilde{A}_{\mu}^{l}, P^{l}\right\} \\
& +i \gamma^{5}\left(\partial_{\mu} P^{l}-\left[\tilde{V}_{\mu}^{l}, P^{l}\right]+\left\{\tilde{A}_{\mu}^{l}, \Sigma^{l}\right\}\right) \\
W_{\mu \nu}^{l}= & \partial_{\mu} \Gamma_{\nu}^{l}-\partial_{\nu} \Gamma_{\mu}^{l}-i\left[\Gamma_{\mu}^{l}, \Gamma_{\nu}^{l}\right] .
\end{aligned}
$$


For the derivative expansion, we again use the momentum averaging trick. It involves $\hat{F}(k+P)$ terms and consequently terms with derivatives of $f(p)$. Here we take the prescription of neglecting them. Hence within this approximation all the fields such as $\mathcal{S}$ will be multiplied by the number $f^{2}(k)$ in the various momentum integrals. The calculation of the quark determinant is formally identical to the previous case:

$$
\begin{aligned}
S_{F} & =-\frac{1}{2} \int \frac{d^{4} x d^{4} k}{(2 \pi)^{4}} \operatorname{tr}\left[\ln \left(G^{-1}\right)+\ln \left(1+G \Pi^{2}+G\left(2 k \cdot \Pi+a_{l}\right)\right)\right], \\
\text { with } \quad G & =\left[k^{2}+f^{2}(k) \mathcal{S}^{2}\right]^{-1}, \quad a_{l}=i \alpha_{\mu \nu} W_{\mu \nu}-\gamma_{\mu} \mathcal{D}_{\mu}^{l} W^{l \dagger} .
\end{aligned}
$$

Coming back to Minkowski space, we obtain a new effective Lagrangian. We omit again in its expression $\rho \pi \pi$ terms and terms involving couplings of pion and axial-vector fields to the derivatives of the scalar field:

$$
\begin{aligned}
\mathcal{L}_{\text {mes }}^{l}= & \frac{1}{2} 2 N_{c} N_{f} I_{2 S}^{l}(\mathcal{S}) \partial^{\mu} \mathcal{S} \partial_{\mu} \mathcal{S}-W^{l}(\mathcal{S})+\frac{m \mathcal{S}}{4 G_{1}} \operatorname{tr}_{f}\left(U+U^{\dagger}-2\right) \\
& +\frac{1}{2} 2 N_{c} \operatorname{tr}_{f}\left[I_{2}^{l}(\mathcal{S}) \mathcal{S}^{2} \partial^{\mu} U \partial_{\mu} U^{\dagger}+4 I_{26}^{l}(\mathcal{S}) \partial^{\mu} P \tilde{A}_{\mu} \Sigma+4 I_{28}^{l}(\mathcal{S}) \tilde{A}^{\mu} \tilde{A}_{\mu} \Sigma^{2}\right] \\
& +\frac{1}{4 G_{2}} \operatorname{tr}_{f}\left(\tilde{V}^{\mu} \tilde{V}_{\mu}+\tilde{A}^{\mu} \tilde{A}_{\mu}\right)-\frac{1}{6} 2 N_{c} N_{f} I_{2 V}(\mathcal{S})\left(\tilde{\Omega}^{\mu \nu} \Omega_{\mu \nu}+\overrightarrow{\tilde{V}}^{\mu \nu} \cdot \overrightarrow{\tilde{V}}_{\mu \nu}+\overrightarrow{\tilde{A}}^{\mu \nu} \cdot \overrightarrow{\tilde{A}}_{\mu \nu}\right) .
\end{aligned}
$$

The new chiral effective potential $W^{l}(\mathcal{S})$ is

$$
W^{l}(\mathcal{S})=-2 N_{c} N_{f} I_{0}^{l}(\mathcal{S})+\frac{(\mathcal{S}-m)^{2}}{2 G_{1}}
$$

The quantity $-I_{0}^{l}(\mathcal{S})$ represents the vacuum energy density associated with the Dirac sea:

$$
\begin{aligned}
I_{0}^{l} & =\int \frac{d^{4} k_{E}}{(2 \pi)^{4}} \ln \left[k_{E}^{2}+f^{4}(k) \mathcal{S}^{2}\right]=\int_{0}^{\infty} \frac{d^{3} k}{(2 \pi)^{3}} E_{k}(\mathcal{S}), \\
E_{k} & \equiv E_{k}(\mathcal{S})=\sqrt{k^{2}+f^{4}(k) \mathcal{S}^{2}},
\end{aligned}
$$

where the second form corresponds to a noncovariant prescription. The various other integrals are

$$
\begin{aligned}
& I_{2 S}^{l}=I_{2}^{l}-2 \mathcal{S}^{2} I_{3}^{l}+2 \mathcal{S}^{4} I_{4}^{l}, \quad I_{2 V}^{l}=I_{2}^{l}+\mathcal{S}^{2} I_{3}^{l}-\frac{1}{2} \mathcal{S}^{4} I_{4}^{l}, \\
& I_{2}^{l}=\int \frac{d^{4} k_{E}}{(2 \pi)^{4}} \frac{f^{4}(k)}{\left[k_{E}^{2}+f^{4}(k) \mathcal{S}^{2}\right]^{2}}=\int_{0}^{\infty} \frac{d^{3} k}{(2 \pi)^{3}} \frac{f^{4}(k)}{4 E_{k}^{3}(\mathcal{S})}, \\
& I_{3}^{l}=\int \frac{d^{4} k_{E}}{(2 \pi)^{4}} \frac{f^{4}(k)}{\left[k_{E}^{2}+f^{4}(k) \mathcal{S}^{2}\right] 3}=\int_{0}^{\infty} \frac{d^{3} k}{(2 \pi)^{3}} \frac{3 f^{4}(k)}{16 E_{k}^{5}(\mathcal{S})}, \\
& I_{4}^{l}=\int \frac{d^{4} k_{E}}{(2 \pi)^{4}} \frac{f^{4}(k)}{\left[k_{E}^{2}+f^{4}(k) \mathcal{S}^{2}\right]^{4}}=\int_{0}^{\infty} \frac{d^{3} k}{(2 \pi)^{3}} \frac{5 f^{4}(k)}{96 E_{k}^{7}(\mathcal{S})}, \\
& I_{26}^{l}=\int \frac{d^{4} k_{E}}{(2 \pi)^{4}} \frac{f^{6}(k)}{\left[k_{E}^{2}+f^{4}(k) \mathcal{S}^{2}\right] 2}=\int_{0}^{\infty} \frac{d^{3} k}{(2 \pi)^{3}} \frac{f^{6}(k)}{4 E_{k}^{3}(\mathcal{S})}, \\
& I_{28}^{l}=\int \frac{d^{4} k_{E}}{(2 \pi)^{4}} \frac{f^{8}(k)}{\left[k_{E}^{2}+f^{4}(k) \mathcal{S}^{2}\right] 2}=\int_{0}^{\infty} \frac{d^{3} k}{(2 \pi)^{3}} \frac{f^{8}(k)}{4 E_{k}^{3}(\mathcal{S})} .
\end{aligned}
$$

Again the pion-axial-vector mixing has to be eliminated. Keeping only the relevant terms for nuclear physics purposes, we obtain the following effective Lagrangian:

$$
\begin{aligned}
\mathcal{L}_{\text {mes }}^{l}= & \frac{1}{2} \frac{I_{2 S}^{l}(\overline{\mathcal{S}})}{I_{2 S}^{l}\left(M_{0}\right)} \partial^{\mu} S \partial \mu S-W\left(\mathcal{S}=g_{0 S} S\right) \\
& +\frac{1}{4} F^{2} M_{\pi}^{2} \frac{\overline{\mathcal{S}}}{M_{0}} \operatorname{tr}_{f}\left(U+U^{\dagger}-2\right) \\
& +\frac{1}{2 F^{2}} \tilde{I}(\overline{\mathcal{S}}) \overline{\mathcal{S}}^{2} \partial^{\mu} \vec{\Phi} \partial^{\mu} \vec{\Phi} \\
& +\frac{1}{2} M_{V}^{2}\left(\omega^{\mu} \omega_{\mu}+\vec{v}^{\mu} \cdot \vec{v}_{\mu}\right)+\frac{1}{2} M_{A}^{2}\left(\vec{a}^{\mu} \cdot \vec{a}_{\mu}\right) \\
& -\frac{1}{4}\left(\omega^{\mu \nu} \omega_{\mu \nu}+\vec{v}^{\mu \nu} \cdot \vec{v}_{\mu \nu}+\vec{a}^{\mu \nu} \cdot \vec{a}_{\mu \nu}\right),
\end{aligned}
$$

where we have introduced the quantities

$$
\begin{aligned}
I^{l}(\overline{\mathcal{S}}) & =2 N_{c} N_{f} I_{2}^{l}(\overline{\mathcal{S}}), \quad \tilde{I}^{l}(\overline{\mathcal{S}})=2 N_{c} N_{f} \tilde{I}_{2}^{l}(\overline{\mathcal{S}}), \\
\tilde{I}^{l}(\overline{\mathcal{S}}) & \equiv 2 N_{c} N_{f} \tilde{I}_{2}^{l}(\overline{\mathcal{S}})=I^{l}(\overline{\mathcal{S}})-\frac{4 G_{2} \overline{\mathcal{S}}^{2} I_{6}^{l 2}(\overline{\mathcal{S}})}{1+4 G_{2} \overline{\mathcal{S}}^{2} I_{8}^{l}(\overline{\mathcal{S}})}, \\
F^{2} & =\tilde{I}^{l}\left(M_{0}\right) M_{0}^{2} .
\end{aligned}
$$

The constant $F$ given above defines the canonical pion field $\Phi$ through $U=\exp (\Phi / F)$. The canonical vector and axialvector fields are defined according to

$$
\begin{aligned}
\tilde{\Omega}^{\mu}=g_{V} \omega^{\mu}, \quad \tilde{V}^{\mu} & =g_{V} v^{\mu}, \\
\tilde{A}^{\mu}+\frac{2 G_{2} \overline{\mathcal{S}}^{2} I_{6}^{l}(\overline{\mathcal{S}})}{1+4 G_{2} \overline{\mathcal{S}}^{2} I_{8}^{l}(\overline{\mathcal{S}})} \partial^{\mu} \Phi & =g_{V} a^{\mu},
\end{aligned}
$$

with

$$
\begin{aligned}
g_{V}^{2} & =\frac{3 / 2}{2 N_{c} N_{f} I_{2 V}^{l}(\overline{\mathcal{S}})}, \quad M_{V}^{2}=\frac{g_{V}^{2}}{G_{2}}, \\
\frac{M_{A}^{2}}{M_{V}^{2}} & =1+4 G_{2} I_{8}^{l}(\overline{\mathcal{S}}) \overline{\mathcal{S}}^{2} .
\end{aligned}
$$

Coming back to the previous form of the Lagrangian [Eq. (47)], the axial weak current, introduced through the replacement 
$f^{2}(k) \tilde{A}_{\mu} \rightarrow f^{2}(k) \tilde{A}_{\mu}+\mathcal{A}_{\mu}$, coupling to the quantity $\partial^{\mu} \Phi$ allows the identification of the pion decay constant parameter $F_{\pi}$ with the parameter $F$ :

$$
F_{\pi}=\frac{I^{l}\left(M_{0}\right) M_{0}^{2}}{F}-\frac{2 I_{6}^{l}\left(M_{0}\right) M_{0}^{2}}{F} \frac{2 G_{2} M_{0}^{2} I_{6}^{l}\left(M_{0}\right)}{1+4 G_{2} M_{0}^{2} I_{8}^{l}\left(M_{0}\right)} \equiv F .
$$

The pion mass parameter keeps its formal expression

$$
M_{\pi}^{2}=\frac{m M_{0}}{G_{1} F_{\pi}^{2}} .
$$

As for the scalar field, the rescaling parameter [similar to Eq. (31)] becomes $g_{0 S}^{2}=\left[2 N_{c} N_{f} I_{2 S}^{l}\left(M_{0}\right)\right]^{-1}$, and the corresponding canonical mass parameter is

$M_{\sigma}^{2}=g_{0 S}^{2}\left(\frac{\partial^{2} W}{\partial \mathcal{S}^{2}}\right)_{\mathcal{S}=M_{0}}=\frac{I_{28}^{l}\left(M_{0}\right)}{I_{2 S}^{l}\left(M_{0}\right)}\left[4 M_{0}^{2}+\left(\frac{\tilde{I}_{2}}{I_{28}}\right)_{\mathrm{vac}} M_{\pi}^{2}\right]$.

Notice that the results differ from the sharp cutoff case, since we now have three different integrals, $I_{2}^{l}, I_{26}^{l}$, and $I_{28}^{l}$, in place of one, $I_{2}$.

We have a priori four parameters: $G_{1}, G_{2}, \Lambda$, and the bare quark mass $m$. We use

$$
\Lambda=1 \mathrm{GeV}, \quad m=3.5 \mathrm{MeV}, \quad G_{1}=7.8 \mathrm{GeV}^{-2},
$$

and we obtain for the vacuum quark mass at zero momentum and the quark condensate

$$
M_{0}=371 \mathrm{MeV} \Rightarrow\langle\bar{q} q\rangle=-(286 \mathrm{MeV})^{3}
$$

The $G_{2}$ parameter constrained to reproduce the vectordominance model (VDM) phenomenology is

$$
\left(G_{2}\right)^{\mathrm{VDM}}=\frac{g_{V}^{2}}{M_{V}^{2}}=\left(\frac{2.65}{0.770}\right)^{2} \mathrm{GeV}^{-2} .
$$

With this value, we obtain for the pion parameters,

$$
G_{2}=\left(G_{2}\right)^{\mathrm{VDM}} \Rightarrow F_{\pi}=91.3 \mathrm{MeV}, \quad M_{\pi}=141.3 \mathrm{MeV} .
$$

However, in nuclear matter calculations, we allow for a small variation of $G_{2}$ :

$G_{2}=0.78\left(G_{2}\right)^{\mathrm{VDM}} \Rightarrow F_{\pi}=93.6 \mathrm{MeV}, \quad M_{\pi}=137.8 \mathrm{MeV}$

With this set of values, the low-momentum mass parameters for $M_{\sigma}$ defined in Eq. (57) and for other quantities are

$M_{\sigma}=923 \mathrm{MeV}, \quad M_{V}=1256 \mathrm{MeV}, \quad M_{A}=1398 \mathrm{MeV}$.

The numerical value of the vacuum scalar coupling constant is $g_{0 S}=5.55$. Notice that the numerical values of these mass parameters and the associated coupling constants are significantly altered by the fact that $I_{2 S, V}$ differ from $I_{2}$. However, the ratios $g_{V} / M_{V}$ and $g_{0 S} / M_{\sigma}=0.006 \mathrm{MeV}^{-1}$, which are the relevant quantities for nuclear matter calculation, are not sensitive to this effect.

It is interesting to derive the potential of the equivalent linear $\sigma$ model. It is obtained through a second-order expansion in $\mathcal{S}^{2}$ of the Dirac sea energy defined in Eqs. (48) and (49) around its vacuum expectation value $M_{0}^{2}$. We recover the usual linear $\sigma$ potential once we introduce a rescaled "effective" scalar field $(S)_{\text {eff }}=\left(F_{\pi} / M_{0}\right) \mathcal{S}$, normalized to $F_{\pi}$ in the vacuum:

$$
\begin{aligned}
W^{L \sigma M}= & \frac{1}{4} \frac{\left(M_{\sigma}^{2}\right)_{\mathrm{eff}}-M_{\pi}^{2}}{2 F_{\pi}^{2}}\left[\left(S^{2}\right)_{\mathrm{eff}}-F_{\pi}^{2} \frac{\left(M_{\sigma}^{2}\right)_{\mathrm{eff}}-3 M_{\pi}^{2}}{\left(M_{\sigma}^{2}\right)_{\mathrm{eff}}-M_{\pi}^{2}}\right]^{2} \\
& -F_{\pi} M_{\pi}^{2}(S)_{\mathrm{eff} .}
\end{aligned}
$$

This potential has the form of the linear $\sigma$ model potential and the parameters, instead of being arbitrary constants, have been dynamically generated. We remind that our field $(S)_{\mathrm{eff}}$ is a chiral invariant, so as to respect all chiral constraints for the mass evolution, while the scalar field of the $\sigma$ model, $\sigma$, is not. The mass associated with this effective scalar field is

$$
\left(M_{\sigma}^{2}\right)_{\mathrm{eff}}=\left[4 M_{0}^{2}\left(\frac{\tilde{I}_{2}}{I_{28}}\right)_{\mathrm{vac}}+M_{\pi}^{2}\right] \Rightarrow\left(M_{\sigma}\right)_{\mathrm{eff}}=659 \mathrm{MeV}
$$

and the corresponding scalar coupling constant is the one of the quark level linear $\sigma$ model $\left(g_{0 S}\right)_{\text {eff }}=M_{0} / F_{\pi}$. Taking the nucleon as a naive juxtaposition of three constituent quarks, its mass evolution at low density goes as follows:

$$
M_{N}^{*} \simeq M_{N}+3\left(g_{0 S}\right)_{\mathrm{eff}}\left[(S)_{\mathrm{eff}}-F_{\pi}\right]
$$

\section{Completion of the model}

To prepare the ground for a quark-diquark model of the nucleon (see next section), we also introduce an interaction in the quark-quark channel. We limit ourselves to the color $\overline{3}$, scalar-isoscalar diquark channel. The corresponding contribution to the NJL interaction is

$$
\mathcal{L}_{\text {diquark }}=\frac{\tilde{G}_{1}}{2}\left(\bar{\psi}_{c} i \gamma_{5} \tau_{2} \beta_{a} \psi\right)\left(\bar{\psi} i \gamma_{5} \tau_{2} \beta_{a} \psi_{c}\right),
$$

where $\beta_{a}=\sqrt{3 / 2} \lambda_{a}(a=2,5,7)$ are color matrices, $\psi_{c}=$ $i \gamma_{2} \psi^{*}$ is the charge conjugate of the quark spinor, and $\tilde{G}_{1}$ is a (positive) coupling constant. It can be generalized to the delocalized version exactly as for the case of the interaction in the $q \bar{q}$ channel. In the presence of the diquark channel, the bosonization procedure can be also done in the presence of diquarks using the Nambu-Gorkov formalism. In the simplest approximation with a constant scalar background field, one obtains the mass and kinetic energy Lagrangian for the scalarisoscalar diquark fields, $\Delta_{S}^{a}$ :

$$
\mathcal{L}_{\text {diquark }}^{l}=\partial^{\mu} \Delta_{S}^{a} \partial_{\mu} \Delta_{S}^{a \dagger}-M_{D}^{2} \Delta_{S}^{a} \Delta_{S}^{a \dagger} .
$$

The diquark mass in a background scalar field $\overline{\mathcal{S}}$ is

$$
M_{D}^{2}(\overline{\mathcal{S}})=\frac{1}{2 N_{c} N_{f} \tilde{G}_{1} I_{2}^{l}(\overline{\mathcal{S}})}-\frac{2 I_{1}^{l}(\overline{\mathcal{S}})}{I_{2}^{l}(\overline{\mathcal{S}})}
$$

We now come to the valence quark sector of the Lagrangian. It also has an explicit delocalized form written below: 


$$
\begin{aligned}
\mathcal{L}_{\mathrm{val}}^{l}(x)= & \bar{\Psi}(x) i \gamma^{\mu} \partial_{\mu} \Psi(x) \\
& -\int d^{4} x_{1} d^{4} x_{2} \bar{\Psi}\left(x_{1}\right) F_{c}\left(x_{1}-x\right)\left[\Sigma+i P \gamma^{5}+\gamma^{\mu}\left(\tilde{V}_{\mu}+\gamma_{5} \tilde{A}_{\mu}\right)\right](x) F_{c}\left(x-x_{2}\right) \Psi\left(x_{2}\right) \\
& -\int d^{4} x_{1} d^{4} x_{2} \bar{\Psi}_{c}\left(x_{1}\right) F_{c}\left(x_{1}-x\right) i \gamma_{5} \tau_{2} \beta_{a} \Delta_{S}^{a \dagger}(x) F_{c}\left(x-x_{2}\right) \Psi\left(x_{2}\right) \\
& -\int d^{4} x_{1} d^{4} x_{2} \bar{\Psi}\left(x_{1}\right) F_{c}\left(x_{1}-x\right) i \gamma_{5} \tau_{2} \beta_{a} \Delta_{S}^{a}(x) F_{c}\left(x-x_{2}\right) \Psi_{c}\left(x_{2}\right) .
\end{aligned}
$$

The full Lagrangian, $\mathcal{L}_{\text {mes }}^{l}+\mathcal{L}_{\text {diquark }}^{l}+\mathcal{L}_{\text {val }}^{l}$, can be utilized to describe the nucleon, generating models from a simple juxtaposition of constituent quarks to more refined ones such as chiral solitons (nucleon bound by the chiral fields) or quarkdiquark models including quark exchange diagrams. However, with these models, nuclear matter still remains unstable. This is the motivation for the next section, where we introduce on top of the effective NJL Lagrangian some confining interaction between quarks or between quark and diquark. If it is done, the momentum of a valence quark inside the nucleon will be limited to $p \approx K_{\text {string }}^{1 / 2} \approx \Lambda_{\mathrm{QCD}} \approx 200 \mathrm{MeV}$, which is much smaller than the scale $\Lambda \approx 1 \mathrm{GeV}$ entering the form factor. In such a case the delocalization effect is essentially not visible, and one can ignore the effect of the form factor on the valence quark dynamics. If we again perform the chiral transformation on quark fields and vector fields, the valence quark sector Lagrangian used in nucleon structure calculation can be safely taken as

$$
\begin{aligned}
\mathcal{L}_{\mathrm{val}} \simeq & \bar{Q}\left[i \gamma^{\mu} \partial_{\mu}-\mathcal{S}-\gamma^{\mu}\left(V_{\mu}+\gamma_{5} A_{\mu}\right)\right] Q \\
& -\bar{Q}_{c} i \gamma_{5} \tau_{2} \beta_{a} \Delta_{S}^{a \dagger} Q-\bar{Q}_{c} i \gamma_{5} \tau_{2} \beta_{a} \Delta_{S}^{a} Q .
\end{aligned}
$$

\section{Concluding remarks on this section}

In the pure NJL picture, at finite baryonic density, the value of the constituent quark mass, which is the expectation value of the scalar field $\mathcal{S}$, is modified. It can be obtained selfconsistently from a gap equation modified by the presence of a Fermi sea. However, in the real world, baryonic matter is not made of independent constituent quarks but of clustered objects, the nucleons. These nucleons are embedded in the scalar background field $\overline{\mathcal{S}}$, and the nuclear medium can be seen a priori as a shifted vacuum. The nucleon mass will depend in some way on the scalar background field, and the energy density of symmetric nuclear matter at the Hartree level reads

$$
\begin{aligned}
\frac{E_{0}}{V}= & \varepsilon_{0}=\int \frac{4 d^{3} p}{(2 \pi)^{3}} \Theta\left(p_{F}-p\right)\left(\sqrt{p^{2}+M_{N}^{2}(\overline{\mathcal{S}})}-\left(M_{N}\right)_{\mathrm{vac}}\right) \\
& +W(\overline{\mathcal{S}})+9 \frac{G_{2}}{2} \rho^{2} .
\end{aligned}
$$

The expectation value for the scalar field is self-consistently obtained by minimization of the energy density,

$$
\frac{\partial \varepsilon_{0}}{\partial \overline{\mathcal{S}}}=0 \Leftrightarrow \frac{\overline{\mathcal{S}}-m}{G_{1}}=-2\langle\bar{q} q\rangle(\overline{\mathcal{S}})-\frac{\partial M_{N}}{\partial \overline{\mathcal{S}}}(\overline{\mathcal{S}}) \rho_{s}^{N}\left(M_{N}(\overline{\mathcal{S}})\right),
$$

which constitutes an in-medium modified gap equation. The connection between the field $\mathcal{S}$, normalized to the quark mass, and the scalar field $s$ used in our previous work [1-3,9] is $\bar{s}=\left(F_{\pi} / M_{0}\right)\left(\overline{\mathcal{S}}-M_{0}\right)$ and $\rho_{s}^{N}\left(M_{N}(\overline{\mathcal{S}})\right)$ is the nucleonic scalar density. The scalar coupling constant of the nucleon to the effective scalar field (which is normalized to $F_{\pi}$ in the vacuum) is

$$
\left(g_{S}\right)_{\mathrm{eff}}(\overline{\mathcal{S}})=\frac{M_{0}}{F_{\pi}}\left(\frac{\partial M_{N}}{\partial \overline{\mathcal{S}}}\right),
$$

which depends crucially on nucleon structure. For instance if the nucleon mass fully originates from confinement (bag models $),\left(\frac{\partial M_{N}}{\partial \bar{S}}\right)=0$, the scalar field just decouples from the nucleon, $\left(g_{S}\right)_{\text {eff }}=0$. In this case there is no shift of the vacuum, and the scalar field is thus an irrelevant concept for nuclear matter studies. On the other extreme if the nucleon mass fully originates from chiral symmetry breaking (naive additive NJL, chiral soliton), then the nucleon mass in the medium is affected by the scalar field associated with the dropping of the chiral condensate. However, in this case, attractive tadpole destroys stability. Only in the case where the nucleon mass has a mixed origin can the scalar background field contribute to the nuclear attraction without destroying the stability and saturation properties. In that case, by rearranging its quark structure linked to the confinement mechanism, the nucleon reacts against the scalar field, generating effectively repulsive three-body forces. The origin of this repulsion lies in the decrease of the scalar coupling constant of the nucleon. In short, a possibly important part of the saturation mechanism is associated with the progressive decoupling of the nucleon from the scalar field associated with the dropping of the chiral condensate.

In the next section, we will introduce nucleon models capable of achieving the balance between large enough attraction and sufficient reaction. Of course, one falls here in the modeling uncertainties. However, we show below that a stringent constraint exists for the numerical value of the scalar nucleon coupling constant which is model dependent, from the value of the free nucleon $\sigma$ commutator.

The pion-nucleon $\sigma$ term is an important piece of experimental information. It is obtained from the FeynmanHellman theorem: $\sigma_{N}=m\left(\partial M_{N} / \partial m\right) \simeq 50 \mathrm{MeV}$. It receives a contribution from the pion cloud, $\sigma_{N}^{\text {(pion cloud) }}$. According to previous works [2,15-17] we expect $\sigma_{N}^{\text {(pioncloud) }} \simeq 20 \mathrm{MeV}$, which corresponds to a pion-cloud self-energy of $-420 \mathrm{MeV}$. For the nonpionic part, an explicit calculation in the NJL model shows that the linear $\sigma$ model result is recovered but with the 
nucleon structure aspect hidden in the scalar coupling constant $g_{\sigma} \equiv\left(g_{S}\right)_{\mathrm{eff}}\left(M_{0}\right)$ :

$$
\sigma_{N \sigma}^{\text {(no pion) }}=F_{\pi} g_{\sigma} \frac{M_{\pi}^{2}}{\left(M_{\sigma}^{2}\right)_{\mathrm{eff}}} .
$$

Its numerical value has to be $\sigma_{N \sigma}^{\text {(no pion) }}=\sigma_{N}-\sigma_{N}^{\text {(pion cloud) }} \simeq$ $50-20 \simeq 30 \mathrm{MeV}$. This separation of the $\sigma$ term into two pieces is quantitatively supported by the lattice study of Leinweber et al. [15] on the nucleon mass evolution with the bare quark mass. In their work the pionic part of this evolution which has a nonanalytical behavior is calculated explicitly and subtracted out. For the rest, an expansion is made in powers of $m_{\pi}^{2}$. The linear term in $m_{\pi}^{2}$ is linked to the nonpionic $\sigma$ commutator, giving a value $\sigma_{N \sigma}^{\text {(no pion) }} \simeq 29 \mathrm{MeV}$, close to our value. With the value $\left(M_{\sigma}\right)_{\text {eff }}=659 \mathrm{MeV}$ given previously, it leads to $g_{\sigma} \simeq 7$.

Concerning the nucleon mass problem, there exist QCD sum rules which link in an approximate way the nucleon mass to the condensate both for a free nucleon [18] and for a bound one $[19,20]$. For a dilute medium, these sum rules lead to the following mass evolution:

$$
\begin{aligned}
\frac{M_{N}^{*}}{M_{N}} \simeq & \frac{\langle\bar{q} q\rangle}{\langle\bar{q} q\rangle_{\mathrm{vac}}}=1-\frac{\sigma_{N} \rho}{F_{\pi}^{2} M_{\pi}^{2}}=1 \\
& \quad-\frac{\left(\sigma_{N \sigma}^{\text {(no pion })}+\sigma_{N}^{\text {(pion cloud) }}\right) \rho}{F_{\pi}^{2} M_{\pi}^{2}} \simeq 1+\frac{\bar{s}}{F_{\pi}}-\frac{\left\langle\Phi^{2}\right\rangle}{2 F_{\pi}^{2}},
\end{aligned}
$$

where the last expression is the one obtained from the condensate evolution in the NJL model. We see that the quark condensate modification receives two contributions, one from the scalar field and one from the pion cloud, reconstituting at low density the full pion nuclear $\sigma$ term.

Our description brings important restrictions to this expression. First the pion-cloud contribution to the $\sigma$ commutator, $\sigma_{N}^{\text {(pion cloud) }}$, contributes to the condensate evolution. It does not contribute to the mass evolution, otherwise chiral constraints would be violated (such as the presence of a term in $m_{\pi}$ in the $N N$ potential, forbidden [21] by chiral symmetry). In fact its influence on the mass vanishes in the chiral limit, and hence it is a small effect which we ignore. Only the scalar piece, $\sigma_{N \sigma}^{\text {(no pion) }}$, should then enter the mass evolution. In a pure chiral theory such as NJL, the low-density expansion of the mass evolution is then

$$
\frac{M_{N}^{*}}{M_{N}} \simeq 1-\frac{\sigma_{N \sigma}^{(\text {no pion })} \rho}{F_{\pi}^{2} M_{\pi}^{2}}
$$

This separation already introduces a model dependence in the prediction of the mass evolution. This expression would hold, for instance, for an assembly of nucleons described as superpositions of constituent quarks or by chiral soliton models. Second, when we introduce confinement, i.e., when we go beyond NJL, the evolution of the nucleon mass with density also depends on the nucleon structure through the value of the coupling constant of the scalar field to the nucleon, $g_{\sigma}$. Indeed, to leading order, we have $M_{N}^{*}=M_{N}+g_{\sigma} \bar{s}$ with $\bar{s}=-g_{\sigma} \rho /\left(M_{\sigma}^{2}\right)_{\mathrm{eff}}$ which gives

$$
\frac{M_{N}^{*}}{M_{N}} \simeq 1-\frac{g_{\sigma} F_{\pi}}{M_{N}} \frac{\sigma_{N \sigma}^{\text {(no pion) }} \rho}{F_{\pi}^{2} M_{\pi}^{2}} \simeq 1-\frac{g_{\sigma}}{10} \frac{\sigma_{N \sigma}^{\text {(no pion) }} \rho}{F_{\pi}^{2} M^{2}} .
$$

In the linear $\sigma$ model where $g_{\sigma}=M_{N} / F_{\pi}=10$, we recover the Ioffe sum rule generalized at finite density just corrected from pionic effects. With confinement, the value of the scalar coupling constant is reduced, and the mass evolution is slower than the condensate one. The suppression of the pionic contribution to the mass evolution further accentuates the difference between the mass and condensate evolutions. For instance at normal nuclear density, the condensate has dropped by $\simeq 30 \%$. With the value $g_{\sigma} \simeq 7$ deduced above, the mass reduction is significantly lower, $\simeq 13 \%$. The challenge is to establish the compatibility between our results and the full QCD sum rules.

\section{EFFECT OF CONFINEMENT: SIMPLE MODELS FOR THE IN-MEDIUM NUCLEON}

We now come to the last point of this paper, namely, the modeling of the nucleon mass origin and the scalar response of the nucleon defined from the second derivative of the nucleon mass with respect to the scalar field:

$$
\kappa_{N S}(\overline{\mathcal{S}})=\frac{\partial^{2} M_{N}}{\partial \bar{s}^{2}}=\frac{M_{0}^{2}}{F_{\pi}^{2}}\left(\frac{\partial^{2} M_{N}}{\partial \overline{\mathcal{S}}^{2}}\right)=\frac{M_{0}}{F_{\pi}}\left(\frac{\partial\left(g_{S}\right)_{\mathrm{eff}}}{\partial \overline{\mathcal{S}}}\right) .
$$

For a nucleon made of the simple adjunction of three NJL constituent quarks (or a NJL quark and a NJL diquark), the scalar coupling constant is independent of the scalar field and there is no scalar response. The importance of the response is related to the respective roles of chiral symmetry breaking and confinement in the generation of the nucleon mass. In the following we will consider nucleons built from NJL quarks of mass $M$ bound by some confining force. The information that we need is contained in the relation between the nucleon mass $M_{N}$ and the NJL mass $M, M_{N}=f(M)$. The scalar coupling constant of the nucleon is related to that of the NJL quarks, which is $M / F_{\pi}$, through $g_{S}=\frac{\partial f}{\partial M} \frac{\partial M}{\partial \bar{s}}$. The next derivative with respect to $\bar{s}$ gives the nucleon scalar response. A nonvanishing value requires $\frac{\partial^{2} f}{\partial^{2} M} \neq 0$ and it entirely arises from confinement. For instance, in soliton models where the quarks are bound only by chiral forces, the nucleon mass is proportional to the NJL mass and the second derivative vanishes. In the following we establish this relation for different models, showing their effect on the saturation properties.

In a previous work [22] we have introduced a model of a nucleon made of three constituent quarks bound together by a confining harmonic force. The magnitude of the scalar response which followed was too small to prevent the collapse of nuclear matter. We will come back later to this type of model. A possibility of improvement is to reduce the relative role of chiral symmetry breaking. This can be achieved by considering a nucleon made of a quark and a sufficiently light diquark to leave enough room for confinement. A practical advantage is that a three-body problem is transformed into a simpler 
two-body problem. Besides this simplification, there are theoretical and phenomenological reasons to favor a quark-diquark model of the nucleon with relatively light scalar-isoscalar diquark. For instance the work of Shuryak et al. on hadronic current-correlation functions based on a random instanton vacuum [23] finds a strong attraction in the scalar-isoscalar channel leading to a diquark with a mass about $400 \mathrm{MeV}$. An axial-vector diquark is also found but with a much larger mass of the order of $900 \mathrm{MeV}$. It is also possible to nicely reproduce the light baryon spectrum [24], while a calculation without diquark correlations predicts an abundance of missing resonances [25].

As discussed in a set of works of Bentz et al. (see Ref. [8] for application to nuclear matter), it is possible from the NJL model to construct a nucleon with a diquark component. Introducing the standard interaction in the diquark channel as discussed previously, one obtains for the mass of the scalar diquark result quoted in Eq. (61). This mass is also medium dependent since it depends on the constituent quark mass. Its vacuum value is strongly sensitive to the value of $\tilde{G}_{1}$ which defines the quark-quark interaction [see Eq. (59)]. For $\tilde{G}_{1}=G_{1}$ it is exactly equal to the pion mass. Here we choose

$$
\tilde{G}_{1}=0.92 G_{1} \Rightarrow M_{D}=398.5 \mathrm{MeV},
$$

which turns out to be nearly equal to the constituent quark mass in agreement with the work of Ref. [23]. In Ref. [8], it was realized that to obtain a scalar susceptibility, and consequently nuclear matter saturation, requires a confinement mechanism. An infrared cutoff $\mu_{R} \simeq 200 \mathrm{MeV}$ was thus introduced in the Schwinger proper time regularization scheme. Such a prescription implies that quarks cannot propagate at a relative distance larger than $1 / \mu_{R}$, hence mimicking a confinement mechanism. Here we propose to incorporate confinement in a more direct way. Since the diquark is in an antitriplet color state, it is physically plausible that a string develops between the quark and the diquark as in a $Q \bar{Q}$ meson. We thus introduce a confining potential between the quark and the diquark:

$$
V(r)=\frac{1}{2} K r^{2} \text {. }
$$

In the nonrelativistic limit, the problem reduces to solving the Schrödinger equation for a particle with reduced mass $\mu$, placed in a harmonic potential. In this limit, the mass of the (in-medium) nucleon is given by:

$$
\begin{aligned}
M_{N}(\overline{\mathcal{S}}) & =M(\overline{\mathcal{S}})+M_{D}(\overline{\mathcal{S}})+\frac{3}{2} \sqrt{\frac{K}{\mu(\overline{\mathcal{S}})}} \\
\text { with } \mu & =\frac{M M_{D}}{M+M_{D}} .
\end{aligned}
$$

We take for the string tension a standard value $K=$ $(290 \mathrm{MeV})^{3}$. We obtain for the vacuum nucleon mass $M_{N}=$ $1304 \mathrm{MeV}$. The nucleon mass origin splits roughly into a chiral symmetry breaking component $(60 \%)$ and a confinement component $(40 \%)$. The vacuum value scalar coupling constant of this nucleon to the effective scalar field is $g_{\sigma} \equiv\left(g_{S}\right)_{\text {eff }}\left(M_{0}\right)=$ 7.14. This leads to the value of the nonpionic piece of the $\sigma$ term: $\sigma_{N \sigma}^{\text {(no pion) }}=30 \mathrm{MeV}$, as was required.

To show that such a model is capable of describing the saturation properties of nuclear matter, we calculate the energy

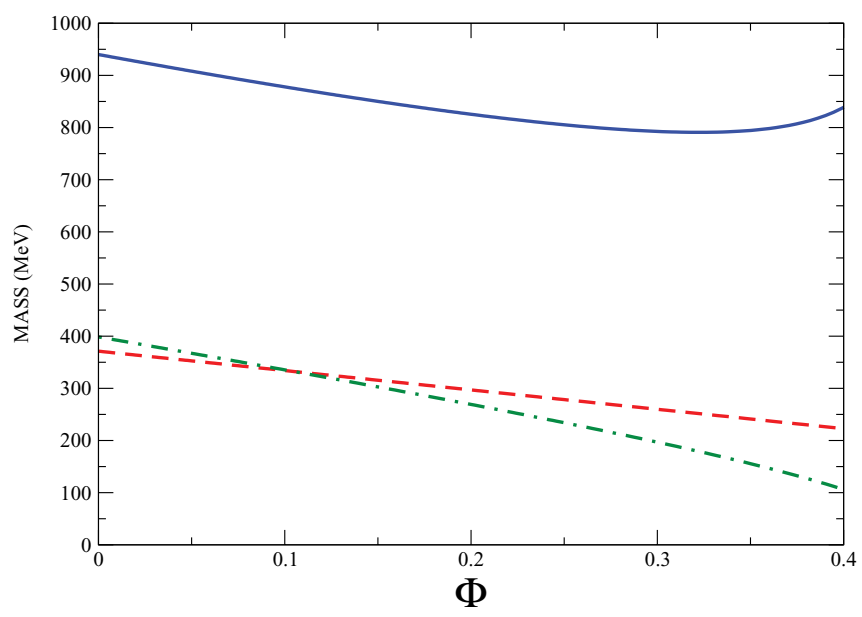

FIG. 1. (Color online) Mass of the quark (dashed line), diquark (dot-dashed line), and nucleon (full line) vs the relative deviation, $\Phi=\left(M_{0}-M\right) / M_{0} \equiv|\bar{s}| / F_{\pi}$, of the scalar field with respect to its vacuum value.

of symmetric nuclear matter in the Hartree approximation, using Eqs. (64) and (65). The resulting curve displays a saturation mechanism driven by the scalar nucleon response $\left[\kappa_{N S}\right.$, proportional to the second derivative of the nucleon mass with respect to $\overline{\mathcal{S}}$, Eq. (70)], which has a positive value. Said differently, the scalar coupling constant, $\partial M_{N} / \partial \overline{\mathcal{S}}$, is a decreasing function of $|\bar{S}|$ or the density. This translates into the fact that the nucleon mass stabilizes or even increases with increasing $|\bar{s}|$ (see Fig. 1). However, the binding is nevertheless not sufficient unless we decrease artificially the vector coupling constant $G_{2}$ at a value much smaller than the VDM result.

To improve the description, although this is not necessarily consistent with our present nucleon model, we add on top of the Hartree mean field result the pion loop (Fock term and correlation energy) contribution obtained in our previous work [2]. Taking the value of $G_{2}$ at the value quoted previously, $G_{2}=0.78\left(G_{2}\right)^{\mathrm{VDM}}$, we obtain a decent saturation curve, shown in Fig. 2. Likely a fully consistent calculation within the model of the pion loop energy would modify the result, but a fine tuning on $G_{2}$ would be presumably sufficient to recover the correct saturation curve. The lesson of this simple model calculation seems to confirm our previous conclusions. The confinement effect (scalar response of the nucleon) is able to stabilize nuclear matter, and the pion loop correlation energy helps to get the correct binding energy.

We have shown that an acceptable quark-diquark model of the nucleon makes plausible the role of the background scalar field in the nuclear binding. It is interesting to investigate if other confining mechanisms can achieve the same result. For this we have also studied models where the nucleon is made of three constituent quarks moving in a mean-field linear confining potential but shifted with a constant attractive potential mimicking short-range attraction:

$$
V=\frac{1+\gamma_{0}}{2}\left(K_{2} r-2 V_{0}\right)
$$




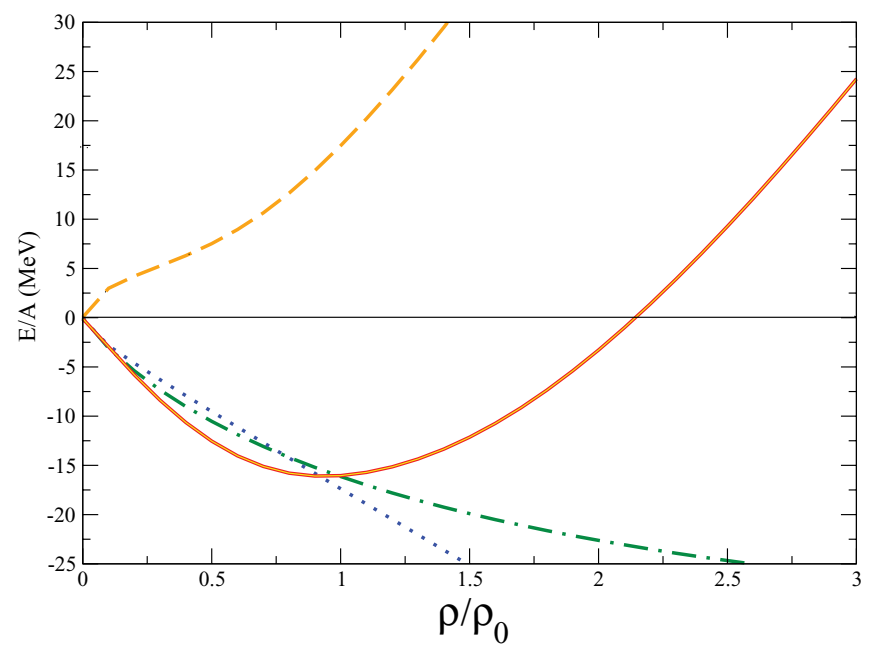

FIG. 2. (Color online) Binding energy of nuclear matter vs nuclear matter density in units of normal density. The full line corresponds to the full result, and the dashed line represents the Hartree result. The dot-dashed line corresponds to the contribution of the Fock term, and the dotted line represents the correlation energy. All the numerical inputs are given in the text.

This model has been successfully utilized for baryon spectroscopy studies by Jena et al. [26]. We do not aim to justify this particular equally mixed scalar and vector confining potentials, the main motivation being the existence of analytical solutions. The energy of the lowest orbit, solution of the Dirac equation, is

$$
E(M)=M-2 V_{0}+\sqrt{K_{2}} x_{q},
$$

with $x_{q}$, solution of

$$
x_{q}^{4}+2 \frac{M-V_{0}}{\sqrt{K_{2}}} x_{q}^{4}-(2.33811)^{3}=0,
$$

and the mass of the in-medium nucleon (in absence of c.m. correction) is $M_{N}(\overline{\mathcal{S}}=M)=3 E(M)$. Hence the quark mass contribution (essentially the chiral symmetry breaking contribution) to the quark orbital energy and then to the nucleon is reduced due to the presence of the attractive shift, $-2 V_{0}$, leaving more room for the confining part. The scalar coupling constant (still omitting c.m. correction) can be written as

$$
\left(g_{S}\right)_{\mathrm{eff}}(\overline{\mathcal{S}})=\frac{M_{0}}{F_{\pi}}\left(\frac{\partial M_{N}}{\partial \overline{\mathcal{S}}}\right) \equiv 3 \frac{M_{0}}{F_{\pi}} q_{s},
$$

where $q_{s}=\int d^{3} r\left(u^{2}-v^{2}\right)(r)$ is the quark scalar charge. We see that the scalar field contribution to the $\sigma$ term is represented by the usual integrated scalar quark density as in bag models. In practice we also include in the numerical calculation the effect of c.m. correction using the results quoted in Ref. [26]. If we take $K_{2}=(300 \mathrm{MeV})^{2}$ and $V_{0}=200 \mathrm{MeV}$, it is possible to obtain a saturation curve, but the saturation has the tendency to come too early. Certainly this point deserves a more detailed study. Here we wish to concentrate on the main result, namely, a decreasing scalar coupling constant when increasing $|\bar{s}|$ as demonstrated by the dashed curve on Fig. 3. We also checked

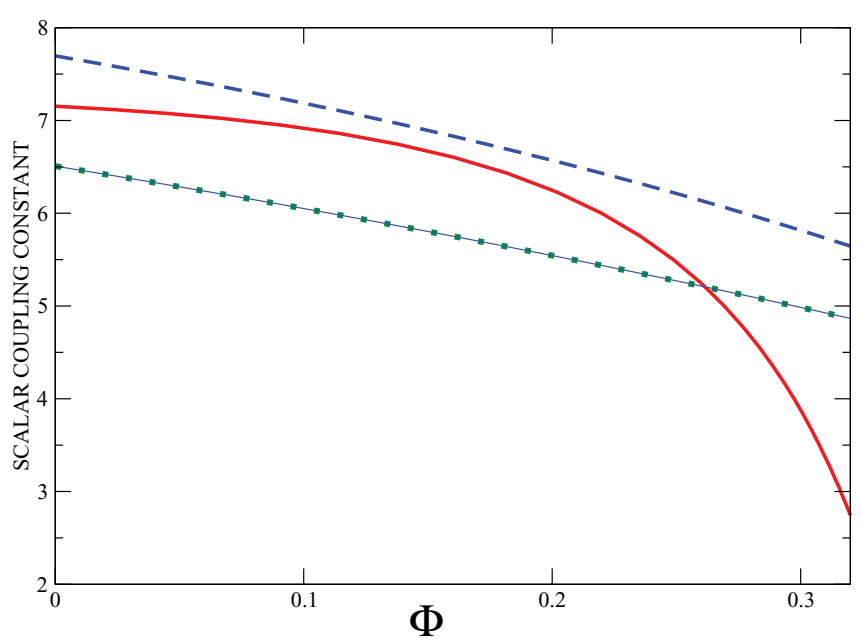

FIG. 3. (Color online) Effective scalar coupling constant vs the relative deviation, $\left(M_{0}-M\right) / M_{0} \equiv|\bar{s}| / F_{\pi}$, of the scalar field with respect to its vacuum value for the linear confining potential (dashed line), the quadratic linear potential (dotted line), and the quark-diquark model (full line).

that replacing the linear potential by a quadratic potential,

$$
V=\frac{1+\gamma_{0}}{2}\left(\frac{1}{2} K_{3} r^{2}-2 V_{0}\right),
$$

with $K_{3}=(300 \mathrm{MeV})^{3}$ and $V_{0}=200 \mathrm{MeV}$, one obtains similar results as depicted in Fig. 3 (dotted curve). It is worthwhile to notice that this model differs from the one used in Ref. [22] by the introduction of the constant attractive shift $-2 V_{0}$. The energy of the lowest orbit is the solution of the equation

$$
E=M-2 V_{0}+\frac{3}{2} \sqrt{\frac{2 K_{3}}{E+M}} .
$$

Again this shift allows us to reinforce the role of confinement in the origin of the nucleon mass.

Also shown on Fig. 3 is the behavior of the scalar coupling constant for the quark-diquark model. In this case, the decrease at low density is less strong, which translates into a softer equation of state. According to a preliminary study based on a variational relativistic calculation, the strong dropping beyond $|\bar{s}| / F_{\pi} \approx 0.2$ (which roughly corresponds to normal density) might be to some extent an artifact of the nonrelativistic approximation.

\section{CONCLUSION}

We have studied the role played by the spontaneous breaking of chiral symmetry in the problem of nuclear binding. The existence of a scalar field linked to the quark condensate emerges in chiral theories such as the NJL one. This field may be at the origin of the masses. This the case in the NJL model or the linear $\sigma$ one. In this case, several things follow naturally. The partial restoration of chiral symmetry in dense matter implies a reduction in magnitude of the condensate and hence of the nucleonic mass, which could a priori account for the nuclear binding; but in this case, 
a tadpole term inherent in these theories destroys stability. A combination with the confining aspects is able to restore stability. Confinement indeed reduces the coupling constant of the scalar field to the nucleon and makes it field dependent. Equivalently it introduces a scalar response a the nucleon to this field in such a way that the nuclear medium reacts against a buildup of the scalar field with increasing density, which helps in the saturation problem. However, confinement should not be the only origin of the nucleon mass, since in this case the scalar background field decouples from the nucleons. It is only in a mixed case, with a simultaneous influence of spontaneous symmetry breaking and confinement, that the scalar field can be an efficient actor in the nuclear saturation problems. We have given examples of nucleonic models where this balance is achieved. They require the role of confinement in the generation of the mass to be sufficient. We have shown how confinement affects the QCD sum rule for the in-medium nucleon mass originally shown to follow the condensate evolution. Our formula shows that the mass evolution is reduced as compared to the condensate one by a factor $r$, ratio of the scalar coupling constants in the presence and in the absence of confinement. In addition, as we pointed out in previous works, only the nonpionic part enters the mass evolution. These combined effects considerably reduce the mass evolution as compared to the condensate one. Nevertheless the remaining effect can be sufficient to make the scalar field of chiral symmetry breaking an important actor in the nuclear binding question.
[1] G. Chanfray and M. Ericson, Eur. Phys. J. A 25, 151 (2005).

[2] G. Chanfray and M. Ericson, Phys. Rev. C 75, 015206 (2007).

[3] E. Massot and G. Chanfray, Phys. Rev. C 80, 015202 (2009).

[4] B. D. Serot and J. D. Walecka, Adv. Nucl. Phys. 16, 1 (1986); Int. J. Mod. Phys. E 16, 15 (1997).

[5] L. S. Celenza et al., Phys. Rev. C 61, 035201 (2000); Ann. Phys. (NY) 241, 1 (1995).

[6] L. S. Celenza, H. Wang, and C. M. Shakin, Phys. Rev. C 63, 025209 (2001).

[7] P. A. M. Guichon, Phys. Lett. B 200, 235 (1988).

[8] W. Bentz and A. W. Thomas, Nucl. Phys. A 696, 138 (2001).

[9] G. Chanfray, M. Ericson, and P. A. M. Guichon, Phys. Rev. C 63, 055202 (2001).

[10] G. Chanfray et al., Eur. Phys. J. A 27, 191 (2006).

[11] W. Bentz, C. Matulla, and H. Baier, Phys. Rev. C 56, 2280 (1997).

[12] Lai-Him Chan, Phys. Rev. Lett. 57, 1199 (1986).

[13] G. Chanfray, M. Ericson, and M. Oertel, Phys. Lett. B 563, 61 (2003).

[14] A. H. Rezaeian, N. R. Walet, and M. C. Birse, Phys. Rev. C 70, 065203 (2004).
[15] D. B. Leinweber, A. W. Thomas, and R. D. Young, Phys. Rev. Lett. 92, 242002 (2004).

[16] I. Jameson, A. W. Thomas, and G. Chanfray, J. Phys. G 18, L159 (1992).

[17] M. C. Birse and J. E. McGovern, Phys. Lett. B 292, 242 (1992); Prog. Theor. Phys. Suppl. 156, 124 (2004); arXiv:nucl-th/0411014.

[18] B. L. Ioffe, Z. Phys. C 18, 67 (1983).

[19] T. D. Cohen, R. J. Furnstahl, and D. K. Griegel, Phys. Rev. Lett. 67, 961 (1991).

[20] P. Finelli, N. Kaiser, D. Vretenar, and W. Weise, Nucl. Phys. A 770, 1 (2006).

[21] M. C. Birse and B. Krippa, Phys. Lett. B 381, 397 (1996).

[22] M. Ericson and G. Chanfray, Energy Phys. J. A 34, 215 (2007).

[23] T. Schafer, E. V. Shuryak, and J. J. M. Verbaarschot, Nucl. Phys. B 412, 143 (1994).

[24] E. Santopinto, Phys. Rev. C 72, 022201(R) (2005).

[25] S. Capstick and N. Isgur, Phys. Rev. D 34, 2809 (1986).

[26] S. N. Jena, M. R. Behera, and S. Panda, Phys. Rev. D 55, 291 (1997). 